\title{
ON LIE ALGEBRAS WITH PRIMITIVE ENVELOPES, SUPPLEMENTS
}

\author{
ALFONS I. OOMS
}

\begin{abstract}
Let $L$ be a finite dimensional Lie algebra over a field $k$ of characteristic zero, $U(L)$ its universal enveloping algebra and $Z(D(L))$ the center of the division ring of quotients of $U(L)$. A number of conditions on $L$ are each shown to be equivalent with the primitive of $U(L)$. Also, a formula is given for the transcendency degree of $Z(D(L))$ over $k$.
\end{abstract}

1. Introduction. The aim of this paper is to establish a necessary and sufficient condition on a finite dimensional Lie algebra $L$ over a field $k$ in order that its universal enveloping algebra $U(L)$ is primitive. This settles a problem raised by Professor Jacobson [6, p. 23]. We may restrict ourselves to the case where $k$ is of characteristic zero, since in characteristic $p \neq 0, U(L)$ is not primitive unless $L=0[6, \mathrm{p}$. 255]. On the other hand, $k$ is not assumed algebraically closed throughout the paper. Let $D(L)$ be the division ring of quotients of $U(L), Z(D(L))$ its center. Let $G \subset$ Aut $L$ be the smallest algebraic group whose Lie algebra $L(G)$ contains ad $L$ (i.e. $L(G)$ is the algebraic hull of ad $L$ in End $L$ ). $G$ is called the adjoint algebraic group of $L$. For each linear functional $f \in L^{*}$ we define $L[f]$ to be the collection of all $x \in L$ such that $f(E x)=0$ for all $E \in L(G) . L[f]$ is a Lie subalgebra of $L$ containing the center of $L$. One verifies that $L[f]$ is an ideal of $L(f)$, where $L(f)$ is the radical of the alternating bilinear form $(x, y) \rightarrow f([x, y])$ defined on $L$. Clearly $L[f]=L(f)$ if $L$ is ad-algebraic. Furthermore, let $K(L)$ be the quotient field of the symmetric algebra $S(L), K(L)^{I}$ the subfield of invariants of $K(L)$.

We can now state the main result.

THEOREM. The following conditions are equivalent:

(1) $L[f]=0$ for some $f \in L^{*}$.

(2) $G$ admits an open dense orbit in $L^{*}$ for its contragredient action on $L^{*}$.

(3) $K(L)^{I}=k$.

(4) $Z(D(L))=k$.

(5) $U(L)$ is primitive.

The proof uses some striking properties of the Dixmier-Duflo map [3, pp. $314-320$ ] as well as some earlier results on the subject [7]. Finally, we shall verify that the number $t=\min _{f \in L^{*}} \operatorname{dim} L[f]$ is equal to the transcendency

Received by the editors August 25, 1975.

AMS (MOS) subject classifications (1970). Primary 17B35; Secondary 16A20.

Key words and phrases. Finite dimensional Lie algebra, universal enveloping algebra, primitive algebra, division ring of quotients.

(1) American Mathematical Society 1976 
degree of $Z(D(L))$ over $k$. This follows directly from the isomorphism that exists between $K(L)^{I}$ and $Z(D(L))$ in the algebraically closed case [8].

2. It is understood that we consider the Zariski topology on $L^{*}$. We denote by $O(f)$ the orbit of $f \in L^{*}$ under the contragredient action of $G$ on $L^{*} . O(f)$ is irreducible (since $G$ is irreducible) and open in its closure [1, p. 98]. Following Dixmier we call $r=\min _{f \in L^{*}} \operatorname{dim} L(f)$ the index of $L$ and $f \in L^{*}$ is called regular if $\operatorname{dim} L(f)=r[3, \mathrm{p} .51]$. It is well known that the set $L_{\mathrm{reg}}^{*}$ of all regular linear functionals is an open dense $G$-stable subset of $L^{*}$. A similar property, concerning the Lie subalgebras $L[f]$, is obtained in the following.

Lemma 1. For all $f \in L^{*}$ we have $\operatorname{dim} L[f]+\operatorname{dim} O(f)=\operatorname{dim} L$. Moreover, the collection $\Omega$ of all $f \in L^{*}$ such that $\operatorname{dim} L[f]=t$ is an open dense, $G$ stable subset of $L^{*}\left(t=\min _{f \in L^{*}} \operatorname{dim} L[f]\right)$.

Proof. If $\left\{x_{1}, \ldots, x_{n}\right\}$ is a basis for $L$ and $\left\{E_{1}, \ldots, E_{m}\right\}$ a basis for $L(G)$, then it is easily seen that

$$
\operatorname{dim} L[f]=\operatorname{dim} L-\operatorname{rank}\left(f\left(E_{i} x_{j}\right)_{i j}\right) .
$$

On the other hand, the stabilizer $S(f)$ of $f \in L^{*}$ is a closed subgroup of $G$ and

$$
\operatorname{dim} O(f)=\operatorname{dim} G-\operatorname{dim} S(f)=\operatorname{dim} L(G)-\operatorname{dim} L(S(f)),
$$

where $L(S(f))$, being the Lie algebra of $S(f)$, is the set of all $E \in L(G)$ such that $f \circ E=0$. By considering the bilinear map $L(G) \times L \rightarrow k$ sending $(E, x)$ into $f(E x)$ we observe that

Hence

$$
\operatorname{dim} L(S(f))=\operatorname{dim} L(G)-\operatorname{rank}\left(f\left(E_{i} x_{j}\right)_{i j}\right) .
$$

$$
\operatorname{dim} O(f)=\operatorname{rank}\left(f\left(E_{i} x_{j}\right)_{i j}\right)=\operatorname{dim} L-\operatorname{dim} L[f] .
$$

This takes care of the first part of the lemma.

In particular,

$$
\max _{f \in L^{*}} \operatorname{dim} O(f)=\operatorname{rank}_{K(L)}\left(\left(E_{i} x_{j}\right)_{i j}\right)=n-t .
$$

Thus $\Omega=\left\{f \in L^{*} \mid \operatorname{rank}\left(f\left(E_{i} x_{j}\right)\right)=n-t\right\}$ and is therefore an open dense subset of $L^{*}$. Being the union of all orbits of maximum dimension, $\Omega$ is also $G$-stable.

The following is a result due to Gabriel [3, p. 159].

THEOREM. Let I be a two-sided ideal of $U(L)$. Then the following conditions are equivalent:

(i) $I$ is absolutely primitive (i.e. $I \otimes k^{\prime}$ is primitive in $U\left(L \otimes k^{\prime}\right)$ for every field extension $k^{\prime}$ of $k$ ).

(ii) There exists an algebraically closed extension $k^{\prime}$ of $k$ such that $I \otimes k^{\prime}$ is primitive in $U\left(L \otimes k^{\prime}\right)$.

(iii) $I$ is primitive and the center of the ring of quotients of $U(L) / I$ reduces to $k$. 
We are now in a position to prove the main criterion.

THEOREM 1. Let $L$ be a Lie algebra over $k$. Then the following conditions are equivalent:

(1) $L[f]=0$ for some $f \in L^{*}$.

(2) $G$ admits an open dense orbit in $L^{*}$ for its contragredient action on $L^{*}$.

(3) $K(L)^{I}=k$.

(4) $Z(D(L))=K$.

(5) $U(L)$ is primitive.

Proof. The equivalence of (1), (3) and (4) has already been shown in [7], as well as the implication (5) $\Rightarrow(1)$. Let us now verify (1) $\Leftrightarrow(2)$. Assume $L[f]=0$ for a suitable $f \in L^{*}$. Then Lemma 1 implies that $\operatorname{dim} O(f)=n$ $=\operatorname{dim} L^{*}$. Consequently $O(f)$ is dense in $L^{*}$. It is even open in $L^{*}$ since $O(f)$ is open in its closure. Conversely, if $O(f)$ is open and dense in $L^{*}$ for some $f \in L^{*}$, then $\operatorname{dim} O(f)=n$ which forces $L[f]=0$ (Lemma 1). Moreover, such an orbit is evidently unique (if $O(h), h \in L^{*}$, is also open, then $O(f) \cap O(h) \neq \varnothing$ and thus $O(f)=O(h))$. Since $O(f)$ is the only orbit of maximum dimension, it follows that $O(f)=\Omega$. Therefore $\Omega \cap L_{\mathrm{reg}}^{*} \neq \varnothing$ implies that $\Omega \subset L_{\text {reg }}^{*}$.

(1) $\Rightarrow(5)$. Let $k^{\prime}$ be the algebraic closure of $k$ and put $L^{\prime}=L \otimes k^{\prime}$. Denote by Prim $\left(U\left(L^{\prime}\right)\right)$ the set of all primitive ideals of $U\left(L^{\prime}\right)$, endowed with the Jacobson topology [6, p. 203] and let $J: L_{\text {reg }}^{\prime *} \rightarrow \operatorname{Prim}\left(U\left(L^{\prime}\right)\right)$ be the DixmierDuflo map which assigns to each regular functional $f \in L^{\prime *}$ a primitive ideal $J(f)$ of $U\left(L^{\prime}\right) . J$ is known to be continuous [3, p. 317] and constant on the orbits lying in $L_{\text {reg }}^{\prime *}$ (i.e. $J(g \cdot f)=J(f)$ for all $g \in G^{\prime}, G^{\prime}$ being the algebraic adjoint of $L^{\prime}$ ) [3, p. 84], [8, p. 394]. Furthermore, if $Q \subset L_{\text {reg }}^{\prime *}$ is dense in $L^{\prime *}$ then $\cap_{f \in Q} J(f)=0[3$, p. 320].

In carrying out the proof of Lemma 1 we came across the formula

$$
t=\min _{f \in L^{*}} \operatorname{dim} L[f]=\operatorname{dim} L-\operatorname{rank}_{K(L)}\left(\left(E_{i} x_{j}\right)_{i j}\right)
$$

whenever $\left\{E_{1}, \ldots, E_{m}\right\}$ is a basis for $L(G)$ and $\left\{x_{1}, \ldots, x_{n}\right\}$ a basis for $L$. Clearly this number $t$ remains unchanged under extension of the base fiejd $k$. So, if $L$ satisfies (1) (i.e. $t=0$ ) the same holds for $L^{\prime}$. Then the foregoing observation shows that there exists an orbit $\Omega^{\prime} \subset L_{\text {reg }}^{\prime *}$ which is open dense in $L^{\prime *}$. Choose $h \in \Omega^{\prime}$. Application of the properties of the map $J$ mentioned above gives

$$
J(h)=\bigcap_{f \in \Omega^{\prime}} J(f)=0 .
$$

Hence the ideal $(0)$ is primitive in $U\left(L^{\prime}\right)$ and by Gabriel's theorem also in $U(L)$. This completes the proof.

REMARK. Because of this theorem, all examples of Lie algebra we have listed in [7] have primitive envelopes, even without the requirement that the base field $k$ is algebraically closed.

Probably the most interesting class of Lie algebras satisfying the conditions of Theorem 1 is formed by the Lie algebras of index 0 , partly because they include all ad-algebraic Lie algebras enjoying these conditions. If $L$ is of index 0 , it admits a linear functional $f \in L^{*}$ such that the alternating bilinear form 
on $L$ sending $(x, y)$ into $f([x, y])$ is nondegenerate, a situation reminiscent of Frobenius algebras in the associative case. In the study of these so called Frobenius Lie algebras, the Lie algebra of all $n \times n$ matrices with entries in $k$ and with last row equal to zero seems to play a significant role. It is an adalgebraic Frobenius Lie algebra satisfying the Gelfand-Kirillov conjecture [4], [7]. However, not all Frobenius Lie algebras are ad-algebraic (example b(iii) of $[7$, p. 497] is not even almost algebraic).

Proposition. Let $L$ be a (finite dimensional) Lie algebra over $k$. If $f, f^{\prime} \in L^{*}$ are such that $L[f]=0=L\left[f^{\prime}\right]$, then $f^{\prime}=g \cdot f$ for some $g \in G, G$ being the adjoint algebraic group of $L$. In particular, in a Frobenius Lie algebra any two regular linear functionals are conjugate by an element of the adjoint algebraic group.

Proof. We know from the proof of Theorem 1 that the set $\Omega$ of all $f \in L^{*}$ such that $L[f]=0$ is an orbit under the action of $G$ on $L^{*}$.

3. Next we want to establish a formula for the transcendency degree tr $\operatorname{deg}_{k}(Z(D(L)))$ of the center $Z(D(L))$ over $k$. For this task we need to recall the following preliminary material.

Let $s$ be the canonical linear isomorphism of $S(L)$ onto $U(L)$, which maps each product $y_{1} \cdots y_{q}, y_{i} \in L$, into $(1 / q !) \sum_{p} y_{p(1)} \cdots y_{p(q)}$ where $p$ ranges over all permutations of $\{1, \ldots, q\}$. Let $\left\{x_{1}, \ldots, x_{n}\right\}$ be a basis of $L$ and $\left\{E_{1}, \ldots, E_{m}\right\}$ a basis for $L(G)$. Then $S(L) \cong k\left[X_{1}, \ldots, X_{n}\right]$ is the direct sum of the subspaces $S^{q}$ of homogeneous polynomials of degree $q$. On the other hand, let $U_{q}, q \geqslant 0$, be the family of subspaces of $U(L)$ which forms the usual increasing filtration of $U(L)$. The associated graded algebra is isomorphic to $S(L)$ by the Poincaré-Birkhoff-Witt theorem. The elements $u \in U_{q} \backslash U_{q-1}$ are said to be of degree $q$ and $[u]=u \bmod U_{q-1}$ is called the leading term of $u$. All nonzero elements $u, v \in U(L)$ satisfy $[u v]=[u][v]$ and $\operatorname{deg}(u v)=\operatorname{deg}(u)$ $+\operatorname{deg}(v)$. If $x=x_{q}+\cdots+x_{0}, x_{q} \neq 0$, is the decomposition of $x \in S(L)$ into homogeneous components $\left(x_{i} \in S^{i}\right)$ then we notice that $[s(x)]=x_{q}$. Every $E \in \operatorname{ad} L$ acts as a derivation in both $K(L)$ and $D(L)$, leaving stable the subspaces $S^{q}$ and $U_{q}$, and commutes with $s$ (i.e. $E s(x)=s(E x)$ for all $x \in S(L)$ ).

In order to proceed we require the following lemmas.

LEMMA 2. $K(L)^{I}$ is generated as a field by elements of the form $x y^{-1} \in K(L)^{I}$, $y \neq 0$, where $x$ and $y$ are homogeneous semi-invariants, i.e. $x \in S^{i}, y \in S^{j}$ for some $i, j \in \mathbf{N}$ and we can find $a \lambda \in(\operatorname{ad} L)^{*}$ such that $E x=\lambda(E) x, E y$ $=\lambda(E) y$ for all $E \in \operatorname{ad} L$.

Proof. Let $u \in K(L)^{I}$. We may write $u=x y^{-1}, y \neq 0$, where $x, y$ $\in S(L)$ are relatively prime. A standard argument shows that there is a $\lambda \in(\operatorname{ad} L)^{*}$ such that $E x=\lambda(E) x, E y=\lambda(E) y$ for all $E \in$ ad $L$. Let $x=x_{p}+\cdots+x_{0}, y=y_{q}+\cdots+y_{0}$ be the decomposition into homogeneous components $\left(x_{i} \in S^{i}, y_{j} \in S^{j}\right)$. Since each $E \in$ ad $L$ maps each $S^{i}$ into itself we see that $E x=E x_{p}+\cdots+E x_{0}$ is the corresponding decomposition of $E x$. It follows that $E x_{i}=\lambda(E) x_{i}$ and similarly $E y_{j}=\lambda(E) y_{j}$ for all $i, j$ and for all $E \in$ ad $L$. Finally, 


$$
u=x y^{-1}=\sum_{i} x_{i} y^{-1}=\sum_{i}\left(\sum_{j} y_{j} x_{i}^{-1}\right)^{-1}
$$

(only those indices $i$ are considered for which $x_{i} \neq 0$ ) where each $y_{j} x_{i}^{-1}$ $\in K(L)^{I}$ satisfies the requirements of the lemma.

LEMMA 3. $\operatorname{tr} \operatorname{deg}_{k}\left(K(L)^{I}\right) \leqslant \operatorname{tr} \operatorname{deg}_{k}(Z(D(L)))$.

Proof. The previous lemma guarantees that we can single out a transcendency basis for $K(L)^{I}$ of the form $x_{1} y_{1}^{-1}, \ldots, x_{t} y_{t}^{-1}, y_{i} \neq 0$, where all $x_{i}, y_{i} \in S(L)$ are homogeneous semi-invariants. Put $u_{i}=s\left(x_{i}\right), v_{i}$ $=s\left(y_{i}\right)$ and $z_{i}=u_{i} v_{i}^{-1}$. We observe that for all $E \in \operatorname{ad} L, E u_{i}=E s\left(x_{i}\right)$ $=s\left(E x_{i}\right)=\lambda(E) s\left(x_{i}\right)=\lambda(E) u_{i}$ and similarly $E v_{i}=\lambda(E) v_{i}$. Consequently, $z_{i} \in Z(D(L))$ since

$$
\begin{aligned}
E z_{i} & =E\left(u_{i} v_{i}^{-1}\right)=\left(E u_{i}-u_{i} v_{i}^{-1} E v_{i}\right) v_{i}^{-1} \\
& =\left(\lambda(E) u_{i}-u_{i} v_{i}^{-1} \lambda(E) v_{i}\right) v_{i}^{-1}=0 \text { for all } E \in \operatorname{ad} L .
\end{aligned}
$$

Clearly, it suffices to show that $z_{1}, \ldots, z_{t}$ are algebraically independent over $k$. Suppose we can find some $a_{q} \in k$, not all zero $\left(q=\left(q_{1}, \ldots, q_{t}\right)\right)$ such that $\sum_{q} a_{q} z_{1}^{q_{1}} \cdots z_{t}^{q_{t}}=0$. Let $m_{i}$ be the largest exponent of $z_{i}$ that appears nontrivially in this sum. Since $u_{i}$ and $v_{i}$ commute with each other we obtain, after multiplication with $v_{1}^{m_{1}} \cdots v_{t}^{m_{t}}$, that

$$
\sum_{q} a_{q} u_{1}^{q_{1}} v_{1}^{m_{1}-q_{1}} \cdots u_{t}^{q_{t}} v_{t}^{m_{t}-q_{t}}=0 \text {. }
$$

Let $m$ be the largest degree (as defined in the preliminaries) of all monomials appearing nontrivially in this sum and let $Q$ be the set of all $q$ 's with $a_{q} \neq 0$ and corresponding with the monomials of degree $m$. Then it follows that

$$
\sum_{q \in Q} a_{q}\left[u_{1}\right]^{q_{1}}\left[v_{1}\right]^{m_{1}-q_{1}} \cdots\left[u_{t}\right]^{q_{t}}\left[v_{t}\right]^{m_{t}-q_{t}}=0 .
$$

After dividing by $\left[v_{1}\right]^{m_{1}} \cdots\left[v_{t}\right]^{m_{t}}$ and taking into account that $\left[u_{i}\right]=\left[s\left(x_{i}\right)\right]$ $=x_{i}$ and $\left[v_{i}\right]=\left[s\left(y_{i}\right)\right]=y_{i}$ we conclude that $\sum_{q \in Q} a_{q}\left(x_{1} y_{1}^{-1}\right)^{q_{1}} \cdots\left(x_{t} y_{t}^{-1}\right)^{q_{t}}$ $=0$ which contradicts our original assumption.

Lemma 4. Let $k^{\prime}$ be an extension field of $k$ and put $L^{\prime}=L \otimes k^{\prime}$. Then $\operatorname{tr} \operatorname{deg}_{k}(Z(D(L))) \leqslant \operatorname{tr} \operatorname{deg}_{k^{\prime}}\left(Z\left(D\left(L^{\prime}\right)\right)\right)$.

Proof. The identification of $U(L) \otimes k^{\prime}$ with $U\left(L^{\prime}\right)$ results in an imbedding of $D(L) \otimes k^{\prime}$ into $D\left(L^{\prime}\right)$ and thus $D(L)$ and $k^{\prime}$ are linearly disjoint in $D\left(L^{\prime}\right)$. Therefore we may consider $Z(D(L)) \otimes k^{\prime} \subset Z\left(D\left(L^{\prime}\right)\right)$. Suppose $z_{1}, \ldots, z_{p}$ $\in Z(D(L))$ are algebraically independent over $k$. This means that the monomials $z_{1}^{n_{1}} \cdots z_{p}^{n_{p}}, n_{i} \in \mathbf{N}$, are linearly independent over $k$. Hence $z_{1}^{n_{1}} \cdots z_{p}^{n_{p}} \otimes 1, n_{i} \in \mathbf{N}$, are linearly independent over $k^{\prime}$. This implies that $z_{1} \otimes 1, \ldots, z_{p} \otimes 1$ are algebraically independent over $k^{\prime}$. The result then follows immediately.

THEOREM 2. Let $L$ be a Lie algebra over $k, G$ its adjoint algebraic group acting on $L^{*}$ and $M$ the largest dimension of all orbits in $L^{*}$. Then $Z(D(L))$ and $(K(L))^{I}$ have the same transcendency degree over $k$, equal to the number $t$ $=\min _{f \in L^{*}} \operatorname{dim} L[f]=\operatorname{dim} L-M$. 
Proof. Let $k^{\prime}$ be the algebraic closure of $k$ and put $L^{\prime}=L \otimes k^{\prime}$. Then the fields $Z\left(D\left(L^{\prime}\right)\right)$ and $K\left(L^{\prime}\right)^{I}$ are $k^{\prime}$-isomorphic [8, p. 401]. This combined with the preceding lemmas yields

$$
\begin{aligned}
\operatorname{tr} \operatorname{deg}_{k}\left(K(L)^{I}\right) & \leqslant \operatorname{tr} \operatorname{deg}_{k}(Z(D(L))) \leqslant \operatorname{tr} \operatorname{deg}_{k^{\prime}}\left(Z\left(D\left(L^{\prime}\right)\right)\right) \\
& =\operatorname{tr} \operatorname{deg}_{k^{\prime}}\left(K\left(L^{\prime}\right)^{I}\right) .
\end{aligned}
$$

On the other hand, $\operatorname{tr} \operatorname{deg}_{k}\left(K(L)^{I}\right)=\operatorname{tr} \operatorname{deg}_{k^{\prime}}\left(K\left(L^{\prime}\right)^{I}\right)$. Indeed, we know that

$$
\operatorname{tr} \operatorname{deg}_{k}\left(K(L)^{I}\right)=\operatorname{dim} L-\operatorname{rank}_{K(L)}\left(\left(E_{i} x_{j}\right)_{i j}\right) \quad \text { [7], }
$$

which we have seen (in the proof of Theorem 1) to be equal to $t$ $=\min _{f \in L^{*}} \operatorname{dim} L[f]=\operatorname{dim} L-M$ and which does not change under field extension. Hence, we may conclude that

$$
\operatorname{tr} \operatorname{deg}_{k}(Z(D(L)))=\operatorname{tr} \operatorname{deg}_{k}\left(K(L)^{I}\right)=t .
$$

REMARK. In case $L$ is ad-algebraic the formula we came across in the preceding discussion simplifies to

$$
\operatorname{tr} \operatorname{deg}_{k}(Z(D(L)))=\operatorname{dim} L-\operatorname{rank}_{K(L)}\left(\left[x_{i}, x_{j}\right]\right)
$$

which is now equal to the index of $L$.

\section{REFERENCES}

An extensive reference list can be found in Dixmier's book on enveloping algebras [3].

1. A. Borel, Linear algebraic groups, Benjamin, New York, 1969. MR 40 \#273.

2. W. Borho, P. Gabriel and R. Rentschler, Primideale in Einhüllenden auflösbarer Liealgebren, Lecture Notes in Math., vol. 357, Springer-Verlag, Berlin and New York, 1973.

3. J. Dixmier, Algèbres enveloppantes, Cahiers Scientifiques, fasc. 37, Gauthier-Villars, Paris, 1974.

4. I. M. Gelfand and A. A. Kirillov, Sur les corps liés aux algèbres enveloppantes des algèbres de Lie, Inst. Hautes Etudes Sci. Publ. Math. No. 31 (1966), 5-19. MR 34 \#731.

5. N. Jacobson, Lie algebras, Interscience Tracts in Pure and Appl. Math., no. 10, Wiley, New York, 1962. MR 26 \# 1345.

6. - Structure of rings, 2nd ed., Amer. Math. Soc. Colloq. Publ., vol. 37, Amer. Math. Soc., Providence, R. I., 1964. MR 36 \#5158.

7. A. I. Ooms, On Lie algebras having a primitive universal enveloping algebra, J. Algebra 32 (1974), 488-500.

8. R. Rentschler and M. Vergne, Sur le semi-centre du corps enveloppant d'une algèbre de Lie, Ann. Sci. Ecole Norm. Sup. (4) 6 (1973), 389-405. MR 50 \# 13177.

Department of Mathematics, Universitair Centrum Limburg, 3610 Diepenbeek, Belgium 